\title{
A New Approach to Polymer Flooding: Effects of Early Polymer Injection and Wettability on Final Oil Recovery
}

\author{
J. L. Juárez-Morejón*, H. Bertin, and A. Omari, University of Bordeaux, and \\ G. Hamon, C. Cottin, D. Morel, C. Romero, and G. Bourdarot, Total
}

\begin{abstract}
Summary
An experimental study of polymer flooding is presented here, focusing on the influence of initial core wettability and flood maturity (volume of water injected before polymer injection) on final oil recovery. Experiments were performed using homogeneous Bentheimer Sandstone samples of similar properties. The cores were oilflooded using mineral oil for water-wet conditions and crude oil (after an aging period) for intermediate-wet conditions; the viscosity ratio between oil and polymer was kept constant in all experiments. Polymer, which is a partially hydrolyzed polyacrylamide (HPAM), was used at a concentration of 2,500 ppm in a moderate-salinity brine. The polymer solution was injected in the core at different waterflood-maturity times [breakthrough (BT) and $0,1,1.75,2.5,4$, and 6.5 pore volumes $(\mathrm{PV})]$.

Coreflood results show that the maturity of polymer injection plays an important role in final oil recovery, regardless of wettability. The waterflood-maturity time 0 PV (polymer injection without initial waterflooding) leads to the best sweep efficiency, whereas final oil production decreases when the polymer-flood maturity is high (late polymer injection after waterflooding). A difference of $15 \%$ in recovery is observed between early polymer flooding $(0 \mathrm{PV})$ and late maturity $(6.5 \mathrm{PV})$. Concerning the effect of wettability, the recovery factor obtained with water-wet cores is always lower (from 10 to $20 \%$, depending on maturity) than the values obtained with intermediatewet cores, raising the importance of correctly restoring core wettability to obtain representative values of polymer incremental recovery. The influence of wettability can be explained by the oil-phase distribution at the pore scale. Considering that the waterflooding period leads to different values of the oil saturation at which polymer flooding starts, we measured the core dispersivity using a tracer method at different states. The two-phase dispersivity decreases when water saturation increases, which is favorable for polymer sweep.

This study shows that in addition to wettability, the maturity of polymer flooding plays a dominant role in oil-displacement efficiency. Final recovery is correlated to the dispersion value at which polymer flooding starts. The highest oil recovery is obtained when the polymer is injected early.
\end{abstract}

\section{Introduction}

Enhanced-oil-recovery (EOR) technology is receiving increasing attention by many companies as a solution to satisfy the global oil demand. One of the most implemented chemical-EOR technologies is the injection of polymer, which has been used successfully for decades.

Among all the polymers proposed for EOR applications, HPAM is the most widely used polymer for polymer floods. HPAM is a viscoelastic polymer; its viscosity varies with shear rate and its rheological behavior depends on molecular weight, hydrolysis degree, concentration, ionic strength of brine, temperature, and $\mathrm{pH}$.

The main objective of polymer flooding is to decrease the water mobility by increasing its viscosity as well as decreasing water relative permeability to improve macroscopic oil-sweep efficiency (Lake 1989; Wassmuth et al. 2007; Koh et al. 2016). This injection can be performed as a secondary polymer flood or after an initial waterflooding period (tertiary polymer flood), when the water cut reaches high values. Secondary and tertiary polymer floods, however, behave differently. Cottin et al. (2014) show a lower remaining oil saturation resulting from secondary polymer floods compared with tertiary polymer injection, as a result of a better sweep efficiency at the pore scale.

The efficiency of a polymer flood depends on several factors that should be considered before starting the injection. Skauge et al. (2014) and Shashvat and Mohanty (2015) showed the importance of the viscosity ratio between an oil and brine/polymer solution for the development of water channels (viscous fingering) and its effect on water BT (WBT) and oil recovery. Their results show that the number of fingers is reduced with an increase in the polymer viscosity, and consequently the sweep area is also increased. A very low concentration of polymer (low viscosity) might in some cases bring an important incremental oil recovery (Levitt et al. 2013). Viscoelastic effects of polymer solutions have also been shown to decrease oil saturation over purely viscous fluids (glycerine). Under the same conditions, Wang et al. (2000), Vermolen et al. (2014), and Qi et al. (2016) conducted a series of corefloods and observed a clear reduction of oil saturation as a result of the viscoelastic effect of the polymer. However, for high-viscosity crude oil, this viscoelastic effect did not improve recovery over polymer floods with low viscoelasticity. The capillary number is another important parameter; Shashvat and Mohanty (2015) showed the effects of flow rate on fingering and oil recovery. At the same viscosity ratio $\left(\mu_{o} / \mu_{w}=1,000\right)$, the fingering effects are more important at high flow rates, causing a reduction of oil recovery. Core permeability (Asghari and Nakutnyy 2008) also affects the oil recovery obtained under similar operational conditions (injection rate and viscosity ratio), and is higher in the case of highpermeability cores than in low-permeability cores. Finally, wettability is another important parameter that plays a key role. Oildisplacement efficiency is controlled by the phase repartition at the pore scale and by the flow instabilities during waterflooding at the core scale. It is known that the remaining oil saturation obtained after waterflooding depends on wettability (Jadhunandan and Morrow 1995); the lowest value is obtained with intermediate-wettability cores, but there is no similar evidence for polymer flooding. Shiran and Skauge (2015) showed the influence of wettability on resistance and the residual factor (mobility ratio). A lower extra oil-recovery factor was obtained as a result of a higher resistance and residual factor in water-wet systems than in intermediate-wet systems.

*Now with CEPSA

Copyright $\odot 2018$ Society of Petroleum Engineers

This paper (SPE 190817) was accepted for presentation at the SPE Europec featured at the 80th EAGE Conference and Exhibition, Copenhagen, 11-14 June 2018, and revised for publication. Original manuscript received for review 1 March 2018. Revised manuscript received for review 12 July 2018 . Paper peer approved 18 July 2018. 
Despite the abundant literature results, some questions still remain:

- When is the best moment to inject polymer during waterflooding?

- What is the influence of wettability on polymer-flooding final oil recovery?

To answer these questions, experimental studies were performed to obtain a better understanding of the polymer behavior in porous media and to optimize the polymer-injection process for different wettability conditions. The objective of this paper is to assess the optimal polymer-injection procedure and to investigate the role of core wettability on final oil recovery.

\section{Experiments}

Materials and Fluids. Porous Media. Cores were extracted from a Bentheimer Sandstone outcrop. Its composition is reported in Table 1 (Peksa et al. 2015). Bentheimer Sandstone is known to be homogeneous and initially water-wet.

\begin{tabular}{ccccccccc}
$\begin{array}{c}\text { Chemical } \\
\text { Composition }\end{array}$ & $\mathrm{SiO}_{2}$ & $\mathrm{Al}_{2} \mathrm{O}_{3}$ & $\mathrm{FeO} / \mathrm{Fe}_{2} \mathrm{O}_{3}$ & $\mathrm{TiO}_{2}$ & $\mathrm{CaO}$ & $\mathrm{MgO}$ & $\mathrm{H}_{2} \mathrm{O}$ & Und. \\
\hline wt $(\%)$ & 86.47 & 7.31 & 1.14 & 0.7 & 1.21 & 1.76 & 1.2 & 0.21 \\
\hline
\end{tabular}

Table 1-Bentheimer Sandstone chemical composition.

The cores are $200 \mathrm{~mm}$ long and have a square cross section of $40 \times 40 \mathrm{~mm}^{2}$. They are prepared between two inlet and outlet plates and wrapped by nonwetting epoxy resin reinforced by glass fiber to ensure a unidirectional flow while allowing a good transparency to gamma ray.

Fluids. The mineral oil was a paraffinic white mineral oil with viscosity $\mu_{o}=200 \mathrm{cp}$ at $T=25^{\circ} \mathrm{C}$. The crude oil used is composed mostly of aliphatic, aromatic, and acyclic hydrocarbons, with viscosity $\mu_{o}=128 \mathrm{cp}$ at $T=60^{\circ} \mathrm{C}$. Synthetic brines are prepared from deionized and degassed water $\left(<1 \mathrm{ppm}\right.$ of $\left.\mathrm{O}_{2}\right)$ by addition of potassium iodide $(53 \mathrm{~g} / \mathrm{L})$ and $\mathrm{MgCl}_{2} \cdot 6 \mathrm{H}_{2} \mathrm{O}(7 \mathrm{~g} / \mathrm{L})$, leading to total dissolved solids $(\mathrm{TDS})=28,288 \mathrm{ppm}$ and $\mathrm{pH}=6.0$. The synthetic brine has a viscosity $\mu_{w}=0.47 \mathrm{cp}$ at $T=60^{\circ} \mathrm{C}$ and $\mu_{w}=1 \mathrm{cp}$ at $T=25^{\circ} \mathrm{C}$. Brine composition was chosen to allow wettability change by the aging process. Considering the work of Buckley et al. (1998), wettability depends on many parameters (crude-oil composition, brine salinity and pH, and solid composition). With our crude oil and porous medium, Bentheimer $\mathrm{SiO}_{2}$, the brine composition (TDS, $\mathrm{pH}$, presence of monovalent $\mathrm{K}^{+}$and divalent $\mathrm{Mg}^{2+}$ ) was designed to modify initial core wettability from water-wet to intermediate-wet. This change of wettability was controlled by the Amott test (Juárez-Morejón et al. 2017). The polymer used in the polymer solution was an HPAM. The following procedural steps were followed to prepare the polymer solution.

1. Prepare a mother solution $(C=5,000 \mathrm{ppm})$.

2. Dilute the mother solution to the required concentration.

3. Pre-degrade the polymer solution. This degradation is achieved mechanically by flowing the polymer solution at a high shear rate several times through a capillary tube connected to a valve. This predegradation is then quantified in terms of viscosity loss.

4. Filter the polymer solution at a very low flow rate (without supplementary mechanical degradation) to remove impurities or microgels. The polymer solution is filtered through a sequence of 8-, 5-, and 1.2- $\mu \mathrm{m}$ filters.

Experimental Setup. The experimental setup is represented in Fig. 1. All corefloods are performed with cores in the horizontal position. Each core has three intermediate pressure taps installed $5.8 \mathrm{~cm}$ apart, and pressure taps are also installed to measure the total pressure drop between inlet and outlet. Brine and polymer solution are injected using a volumetric pump, while oil is stored in a vertical cell and displaced from the bottom using a piston pump. Oil is filtered $(0.45 \mu \mathrm{m})$ before being injected in the core to avoid any plugging. Effluents are collected with a fraction collector or a graduated burette. An online densimeter can be used for miscible displacement (dispersion experiments). Experiments are performed at $T=60^{\circ} \mathrm{C}$ when using the crude oil and $T=25^{\circ} \mathrm{C}$ for mineral oil.

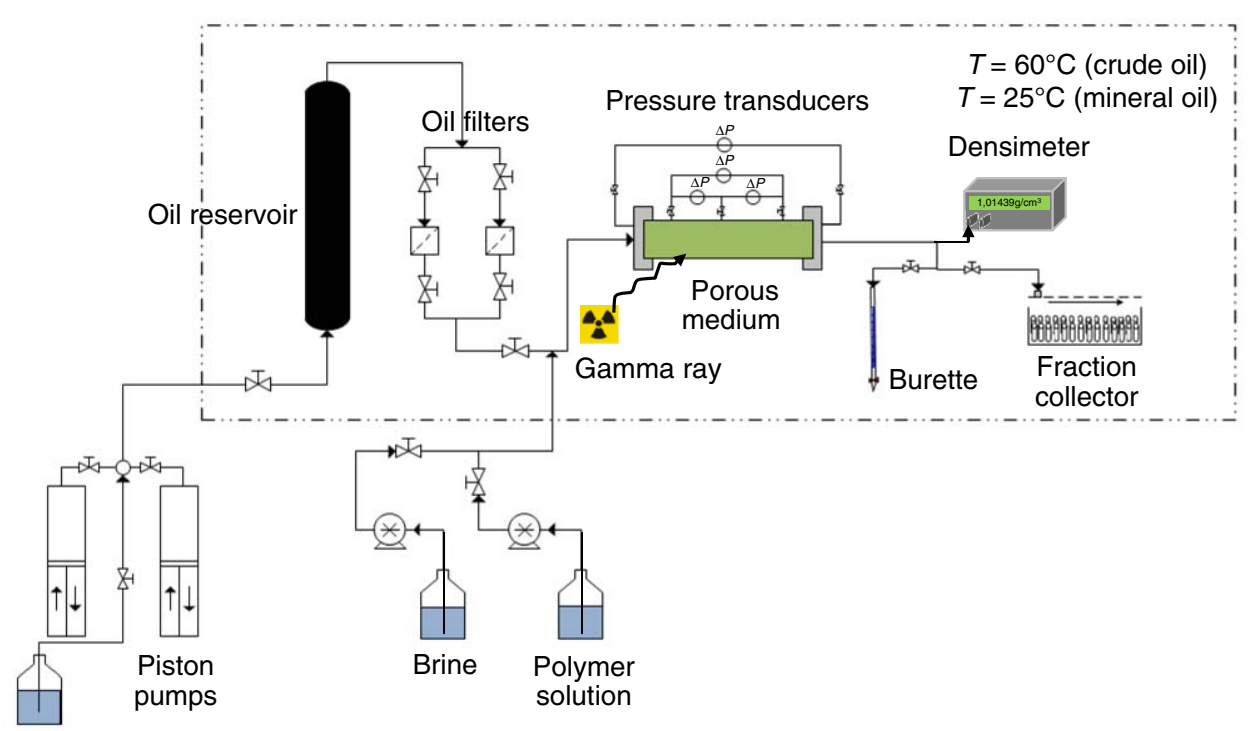

Fig. 1-Experimental setup. 
Experimental Procedure. The experimental procedure used for water-wet and intermediate-wet cores is similar in all aspects except for an aging period, which is set at the end of the initial drainage with crude oil.

1. After core preparation and saturation, porosity is measured by weighing and gamma ray attenuation, while permeability is determined from Darcy's law by applying different flow rates and measuring the corresponding pressure drop.

2. A dispersion test is performed at $T=25^{\circ} \mathrm{C}$ and $Q=0.45 \mathrm{~cm}^{3} / \mathrm{min}$ by injection of a reduced-salinity brine, while density variation is measured continuously. The initial brine is then injected.

3. Water is drained out by oil injection at constant flow rate of $15 \mathrm{~cm}^{3} / \mathrm{h}$ until reaching the irreducible water saturation $\left(S_{w i}\right)$. The oil permeability $\left(k_{o} \text { at } S_{w i}\right)_{1}$ is then measured by increasing the flow rate and measuring the pressure drop.

4. When using the crude oil, the core is aged during a period varying from 14 to 47 days at $T=60^{\circ} \mathrm{C}$ to modify the initial wettability of the porous medium. Relative permeability to oil at $S_{w i}\left(k_{o} \text { at } S_{w i}\right)_{2}$ is measured at the end of aging. (This step is not observed when dealing with mineral oil.)

5. This step corresponds to oil displacement by the aqueous phase (brine followed by polymer solution at different waterfloodmaturity times: $0 \mathrm{PV}, \mathrm{BT}$, and 1, 1.75, 2.5, 4, and 6.5 PV). Oil recovery and pressure drop are measured continuously.

6. When oil production has ended and pressure drop is constant, the aqueous-phase flow rate is increased to minimize the effect of capillary end effects. $S_{o r}$ is measured at this stage by volume balance and by gamma ray attenuation. It is noted that when water injection does not reach $S_{\text {or }}$, the term "remaining oil saturation" will be used.

7. The brine is injected during at very low flow rate $\left(0.25 \mathrm{~cm}^{3} / \mathrm{min}\right)$ to remove any unadsorbed polymer. By then applying different flow rates and measuring the corresponding pressure drop, we obtain the water permeability $\left(k_{w}\right.$ at $\left.S_{o r}\right)$ with a polymer layer adsorbed and $S_{\text {or }}$.

8. A dispersion test is then performed by injection of a reduced-salinity brine while measuring the effluent density continuously to determine the $S_{\text {or }}$ or remaining oil saturation and its corresponding dispersion coefficient.

\section{Experimental Results and Discussion}

Polymer Solution. The polymer concentration selected for our study was $2,500 \mathrm{ppm}$. The mechanical-degradation process involves flowing the solution at a high flow rate $\left(Q=20 \mathrm{~cm}^{3} / \mathrm{min}, \dot{\gamma}=27,135\right.$ seconds $\left.{ }^{-1}\right)$ several times in a capillary tube $(0.5-\mathrm{mm}$ diameter, 2.17-m length). The viscosity of the polymer solution is measured after each flow to evaluate the viscosity loss caused by mechanical degradation. Experimental results are presented in Figs. 2 and 3. The plateau viscosity of the final polymer solution is $\mu_{p}=11.2 \mathrm{cp}$ at $T=60^{\circ} \mathrm{C}$ after 10 flows in the capillary tube and $\mu_{p}=22.4 \mathrm{cp}$ at $T=25^{\circ} \mathrm{C}$ after one flow in the capillary tube.

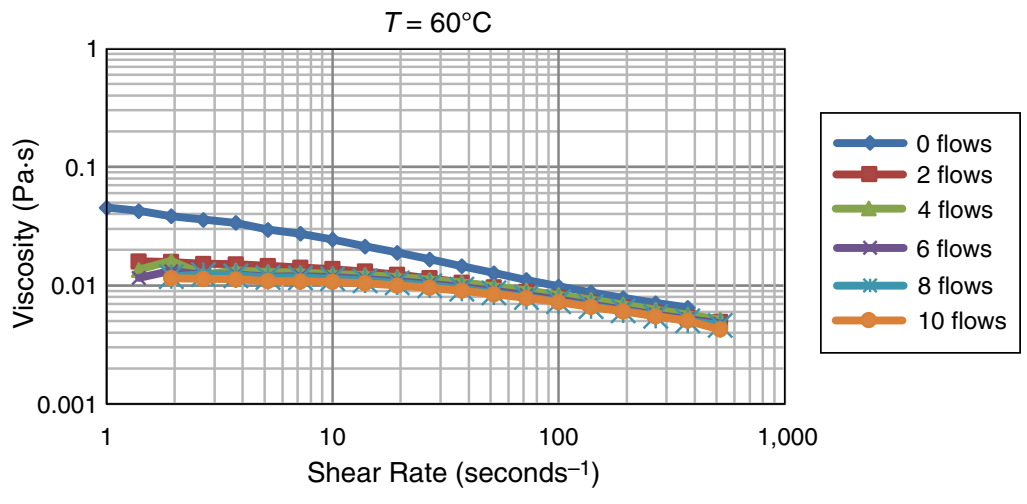

Fig. 2-Variation of the viscosity at $C=2,500 \mathrm{ppm}$. Different flows for a shear rate of 27,135 seconds $^{-1}$ at $T=60^{\circ} \mathrm{C}$.

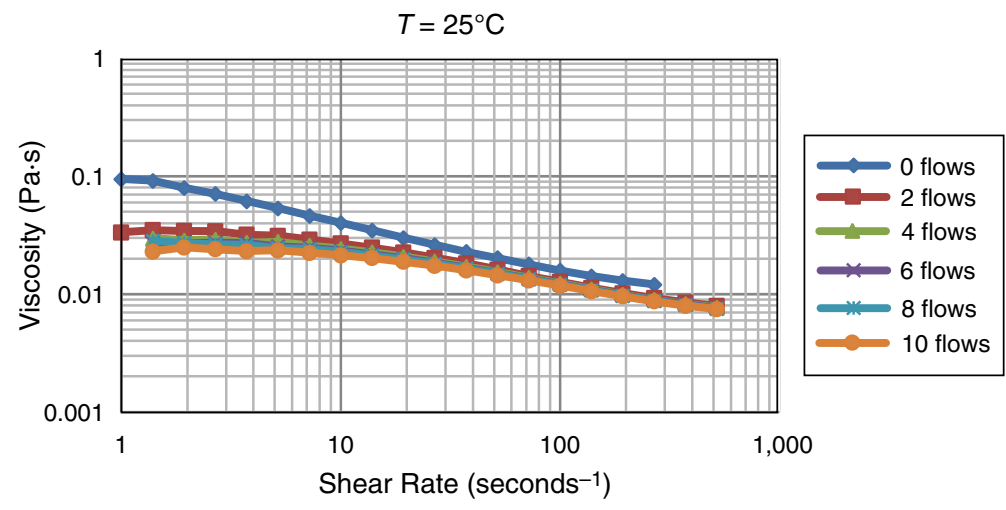

Fig. 3-Variation of the viscosity at $C=2,500 \mathrm{ppm}$. Different flows for a shear rate of $27,135 \operatorname{seconds}^{-1}$ at $T=25^{\circ} \mathrm{C}$.

Wettability Measurement. Cylindrical cores (38-mm diameter, 60-mm length) of Bentheimer Sandstone were considered to evaluate the change of wettability following the Amott test. The experimental procedure to saturate and age the core is similar to the one presented previously. The measured wettability index was $I_{w}=0.075$ (Juárez-Morejón et al. 2017), showing an intermediate wettability of the cores after the aging process. 
Corefloods. All of the 16 corefloods were performed at the same viscosity ratio $\left(\mu_{o} / \mu_{w}=10\right)$ and the same value of capillary number $\left(\mathrm{Ca}=10^{-6}\right)$, which is defined as

$$
C a=\mu \nu / \sigma
$$

where $\mu_{w}$ is the viscosity of the displacing fluid (water or polymer solution), $v$ is the interstitial velocity, and $\sigma$ is the interfacial tension.

The polymer solution is injected at constant flow rate $\left(Q_{w}=0.04 \mathrm{~cm}^{3} / \mathrm{min}\right)$ at different waterflood-maturity times $(0 \mathrm{PV}$, BT, and $1,1.75,2.5,4$, and $6.5 \mathrm{PV})$. The waterfloods were performed at constant flow rate $\left(Q_{w}=0.75 \mathrm{~cm}^{3} / \mathrm{min}\right)$ and the same capillary number. Flow-step-rate increases are performed at the end of the polymer injection to check oil accumulation caused by capillary end effect. Experimental results are reported in Table 2, where every experiment is described by certain codes. WF/PF means waterflood/ polymer flood, followed by the injection-waterflood-maturity time in parentheses; in $0 \mathrm{PV}$, for example, the polymer is injected without previous waterflood. IW means intermediate-wet, and WW means water-wet. Lower-case letters "a" through "e" serve to depict duplicate experiments.

\begin{tabular}{|c|c|c|c|c|c|c|c|c|c|c|c|}
\hline Experiment & $k_{w}\left(\mathrm{~m}^{2}\right)$ & $\phi$ & $S_{w i}$ & $\begin{array}{l}\text { OOIP } \\
\left(\mathrm{cm}^{3}\right)\end{array}$ & $\begin{array}{c}\left(k_{o} \text { at } S_{w i}\right)_{1} \\
\text { Before Aging } \\
\left(\mathrm{m}^{2}\right)\end{array}$ & $\begin{array}{l}\text { Aging } \\
\text { (days) }\end{array}$ & $\begin{array}{c}\left(k_{o} \text { at } S_{w i}\right)_{2} \\
\text { Before Aging } \\
\left(\mathrm{m}^{2}\right)\end{array}$ & $\begin{array}{c}S_{o r} \\
\text { Gamma } \\
\text { Ray }\end{array}$ & $\begin{array}{c}S_{o r} \\
\text { Dispersion } \\
\text { Test }\end{array}$ & $\begin{array}{c}S_{o r} \\
\text { Mass } \\
\text { Balance }\end{array}$ & $\begin{array}{c}\left(k_{w} \text { at } S_{o r}\right)_{2} \\
\left(\mathrm{~m}^{2}\right)\end{array}$ \\
\hline WF/PF (0 PV) IWa & $2.05 \times 10^{-12}$ & 0.244 & 0.126 & 68.11 & $1.75 \times 10^{-12}$ & 22 & $1.89 \times 10^{-12}$ & 0.311 & 0.290 & 0.271 & $1.93 \times 10^{-13}$ \\
\hline WF/PF (0 PV) IWb & $2.15 \times 10^{-12}$ & 0.241 & 0.119 & 68.03 & $2.20 \times 10^{-12}$ & 30 & $1.97 \times 10^{-12}$ & 0.329 & 0.340 & 0.300 & $1.92 \times 10^{-13}$ \\
\hline WF/PF (0 PV) IWc & $1.47 \times 10^{-12}$ & 0.246 & 0.142 & 67.61 & $2.22 \times 10^{-12}$ & 16 & $2.06 \times 10^{-12}$ & 0.293 & 0.290 & 0.273 & $1.33 \times 10^{-13}$ \\
\hline WF/PF (0 PV) IWd & $1.17 \times 10^{-12}$ & 0.240 & 0.128 & 66.9 & $1.78 \times 10^{-12}$ & 35 & $2.03 \times 10^{-12}$ & 0.327 & 0.360 & 0.288 & $1.29 \times 10^{-13}$ \\
\hline WF/PF (0 PV) IWe & $1.60 \times 10^{-12}$ & 0.254 & 0.111 & 71.43 & $2.19 \times 10^{-12}$ & 27 & $2.55 \times 10^{-12}$ & 0.293 & 0.324 & 0.284 & $1.80 \times 10^{-13}$ \\
\hline WF/PF (BT) IWa & $1.15 \times 10^{-12}$ & 0.241 & 0.123 & 67.5 & $1.89 \times 10^{-12}$ & 32 & $1.24 \times 10^{-12}$ & 0.256 & 0.299 & 0.249 & $1.03 \times 10^{-13}$ \\
\hline WF/PF (BT) IWb & $1.32 \times 10^{-12}$ & 0.234 & 0.127 & 65.5 & $1.95 \times 10^{-12}$ & 14 & $2.01 \times 10^{-12}$ & 0.255 & 0.251 & 0.241 & $1.01 \times 10^{-13}$ \\
\hline WF/PF (1 PV) IWa & $1.75 \times 10^{-12}$ & 0.241 & 0.133 & 66.75 & $2.45 \times 10^{-12}$ & 14 & $2.62 \times 10^{-12}$ & 0.252 & 0.300 & 0.293 & $1.22 \times 10^{-13}$ \\
\hline WF/PF (1 PV) IWb & $2.40 \times 10^{-12}$ & 0.264 & 0.146 & 69.27 & $3.16 \times 10^{-12}$ & 41 & $2.48 \times 10^{-12}$ & 0.292 & 0.293 & 0.299 & $3.39 \times 10^{-14}$ \\
\hline WF/PF (1.75 PV) IW & $1.91 \times 10^{-12}$ & 0.247 & 0.153 & 66.9 & $2.09 \times 10^{-12}$ & 40 & $1.83 \times 10^{-12}$ & 0.309 & 0.338 & 0.309 & $1.48 \times 10^{-13}$ \\
\hline WF/PF (2.5 PV) IW & $1.46 \times 10^{-12}$ & 0.238 & 0.132 & 66 & $2.22 \times 10^{-12}$ & 19 & $1.64 \times 10^{-12}$ & 0.335 & 0.338 & 0.373 & $6.18 \times 10^{-14}$ \\
\hline WF/PF (4 PV) IW & $2.22 \times 10^{-12}$ & 0.249 & 0.142 & 68.36 & $3.90 \times 10^{-12}$ & 47 & $3.91 \times 10^{-12}$ & 0.337 & 0.336 & 0.343 & $3.20 \times 10^{-13}$ \\
\hline WF/PF (6.5 PV) IW & $2.22 \times 10^{-12}$ & 0.234 & 0.147 & 63.9 & $3.36 \times 10^{-12}$ & 27 & $3.05 \times 10^{-12}$ & 0.352 & 0.306 & 0.375 & $4.35 \times 10^{-13}$ \\
\hline WF/PF (0 PV) WW & $2.04 \times 10^{-12}$ & 0.249 & 0.147 & 68 & $2.88 \times 10^{-12}$ & - & - & 0.353 & 0.376 & 0.358 & $1.63 \times 10^{-13}$ \\
\hline WF/PF (1 PV) WW & $3.64 \times 10^{-12}$ & 0.253 & 0.140 & 69.7 & $3.87 \times 10^{-12}$ & - & - & 0.437 & 0.455 & 0.452 & $7.83 \times 10^{-14}$ \\
\hline WF/PF (BT) WW & $2.22 \times 10^{-12}$ & 0.251 & 0.131 & 69.9 & $2.06 \times 10^{-12}$ & - & - & 0.365 & 0.404 & 0.407 & $9.45 \times 10^{-14}$ \\
\hline
\end{tabular}

Table 2-Coreflood data. IW = intermediate-wet; $\mathrm{WW}=$ water-wet.

WF/PF (0 PV) Polymer Injection. Intermediate Wettability. Oil recoveries vs. injected volumes for the five experiments, WF/PF (0 PV) IWa through WF/PF (0 PV) IWe, are plotted in Fig. 4. Polymer BTs (PBTs) occur at the same time (0.44 PV) for all cases and are followed by a production tail during $2.5 \mathrm{PV}$, leading to a final recovery of approximately $66 \%$. The oil-production increment after the polymer-flow-rate increase is approximately $2 \%$, showing that the end effect is negligible. This reproducible behavior is typical of intermediate-wettability coreflooding. The $S_{o r}$ and remaining-oil-saturation values, measured by different techniques, are very close (see Table 2).

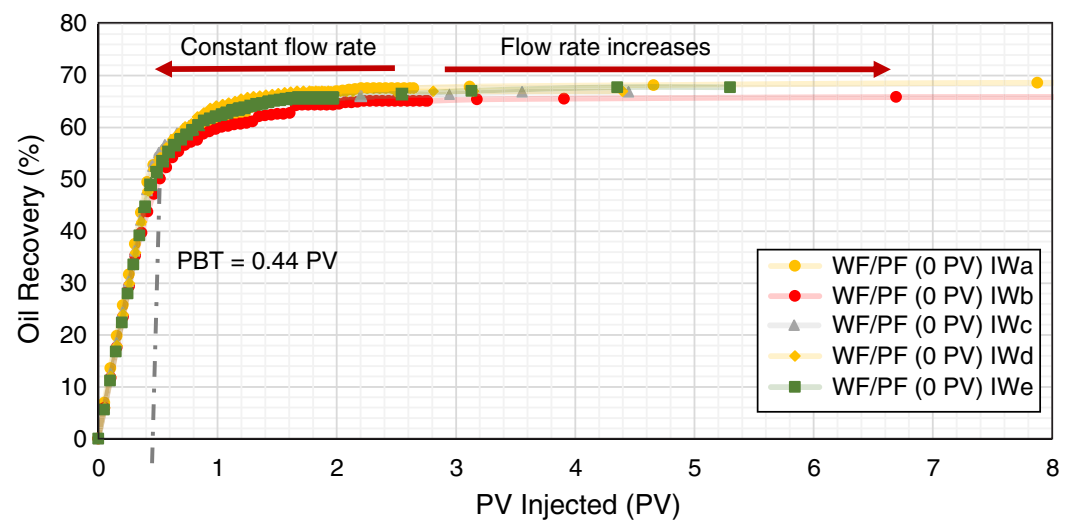

Fig. 4-Oil-recovery curves of five experiments performed in WF/PF (0 PV), with intermediate wettability. PBT = polymer breakthrough.

Fig. 5 plots the oil recovery and pressure drop at different core locations. We observe a progressive diminution of the pressure until PBT of approximately 0.46 . After BT, the pressure remains nearly stable. After 1.6 PV of polymer injection, the oil recovery reached $65 \%$ of original oil in place (OOIP), with the water cut approaching 95\%. The endpoint oil recovery at constant flow was $67.5 \%$ (water cut of $100 \%)$. 


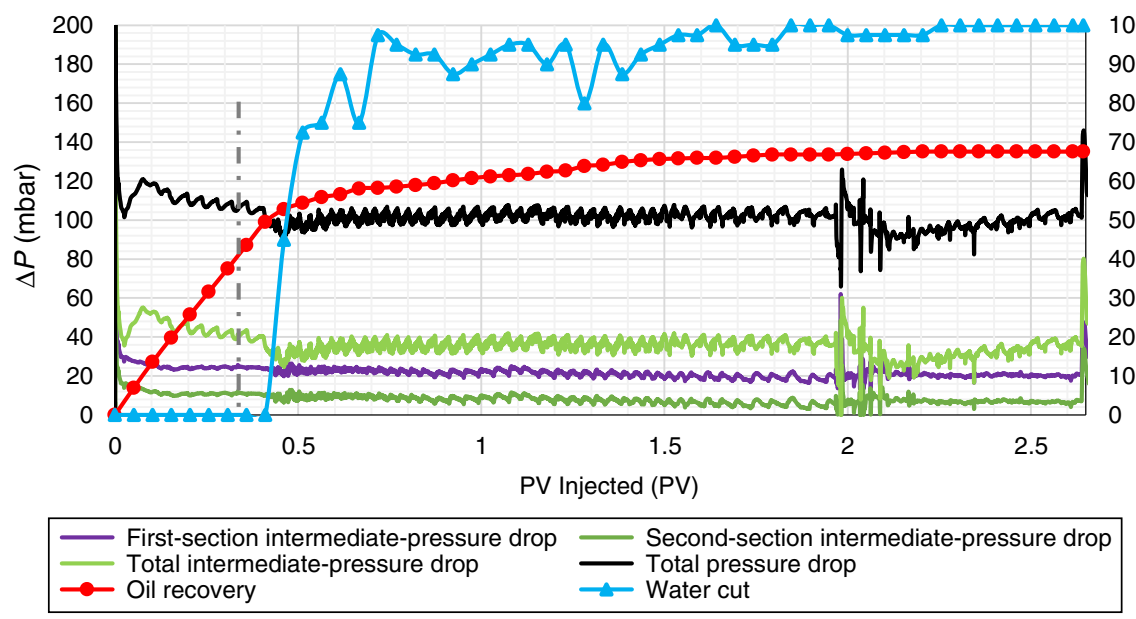

Fig. 5-Pressure drop of WF/PF (O PV) IWa at constant flow rate.

Water-Wet Condition. Oil recovery for the secondary water-wet experiment is presented in Fig. 6. PBT occurs after 0.5 PV and is followed by a quasiplateau of production $(R=58 \%)$. The oil-production increment after flow-rate increase is zero, showing again that the end effect is negligible. This behavior is typical of a water-wet coreflooding.

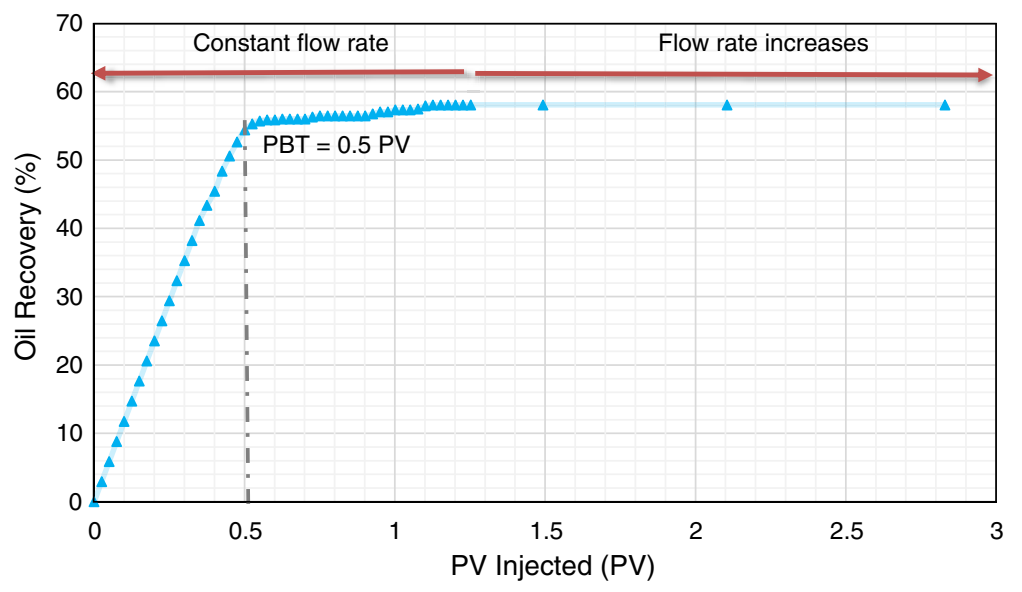

Fig. 6-Oil-recovery curve for a WF/PF (0 PV) water-wet core.

From Figs. 4 and 6, two distinct behaviors are observed depending on the core wettability: After BT, there is little oil production in a water-wet core, but significant production in an intermediate-wet core. This indicates the importance of the location of the two fluids in the porous medium at the moment of polymer injection. When the rock is water-wet, there is a tendency for water to occupy the small pores and to contact the majority of the rock surface. For an intermediate-wet core, the small pores remain water-wet, and water is displaced by oil in the large pores. The surface of the rock is preferentially in contact with oil (Anderson 1986).

Polymer Injection After Initial Waterflooding. Intermediate Wettability. Oil recoveries vs. injected PV for the eight experiments performed in intermediate wettability are plotted in Fig. 7. WBTs occur at $0.16 \mathrm{PV}$ for all the waterflooding, earlier than in WF/PF (0 PV) conditions as a result of an unfavorable water/oil-viscosity ratio ( $\mu_{o} / \mu_{w} \approx 240$ ) leading to a recovery of approximately $19 \%$. Polymer injection is then started at different waterflood-maturity times (BT and 1, 1.75, 2.5, 4, and 6.5 PV). When polymer is injected, we observe a fast pressure increase because of a more-favorable viscosity ratio $\left(\mu_{o} / \mu_{w}=10\right)$ and oil mobilization. When polymer solution is injected at the WBT maturity time, we observe an incremental oil production of $52 \%$. When polymer injection is started at a longer waterflood-maturity time (1 to $6 \mathrm{PV})$, the incremental oil production after waterflooding is much less $(31$ to $20 \%)$.

These results show a significant effect on the final oil recovery for the moment polymer injection is started (maturity). Oil recovery is increased when polymer injection is started the earliest, after WBT. This result will be discussed later, considering the saturation value and the dispersion coefficient.

Fig. 8 shows the polymer injection at concentration of 2,500 ppm into the core after the initial waterflood (1 PV) in intermediate-wet conditions. The injections of the polymer and brine were performed at the same capillary number. An incremental $29 \%$ of OOIP was produced after $1 \mathrm{PV}$ of polymer injection. After $2 \mathrm{PV}$ of polymer injection (water cut $=100 \%$ ), the flow rate is increased to avoid capillary end effects. The oil-production increment after the polymer-flow-rate increase is negligible. At the end of the polymer slug, a total of $66 \%$ of OOIP had been recovered.

Water-Wet Conditions. Two polymer floodings (at waterflood-maturity times BT and $1 \mathrm{PV}$ ) were performed with water-wet cores (Fig. 9). For both cases, the WBT was observed 0.3 PV later than in the intermediate-wet case presented in Fig. 7. In the case of 1-PV maturity time, there is almost no oil production after WBT, and oil production starts again when polymer injection is started. However, the incremental oil production is less than that observed when polymer injection is performed at the same times as the WBT. This behavior is similar to that observed for the intermediate-wet case, but the final oil recovery is approximately $20 \%$ less. 


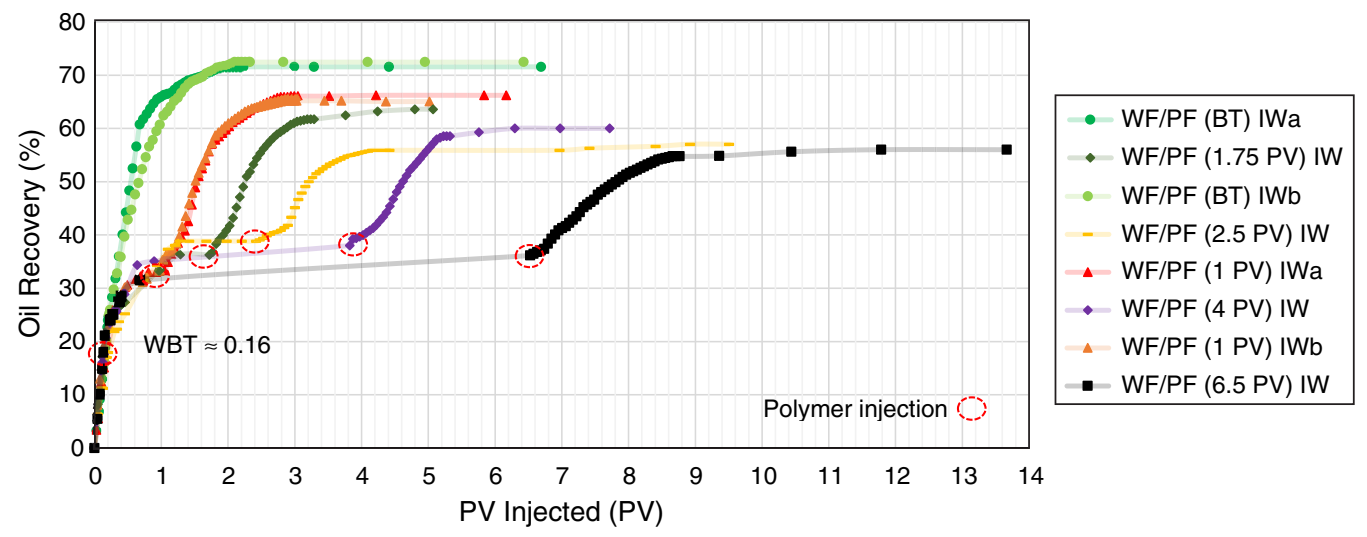

Fig. 7-Oil-recovery curves of eight experiments performed at different waterflood-maturity times with intermediate wettability.

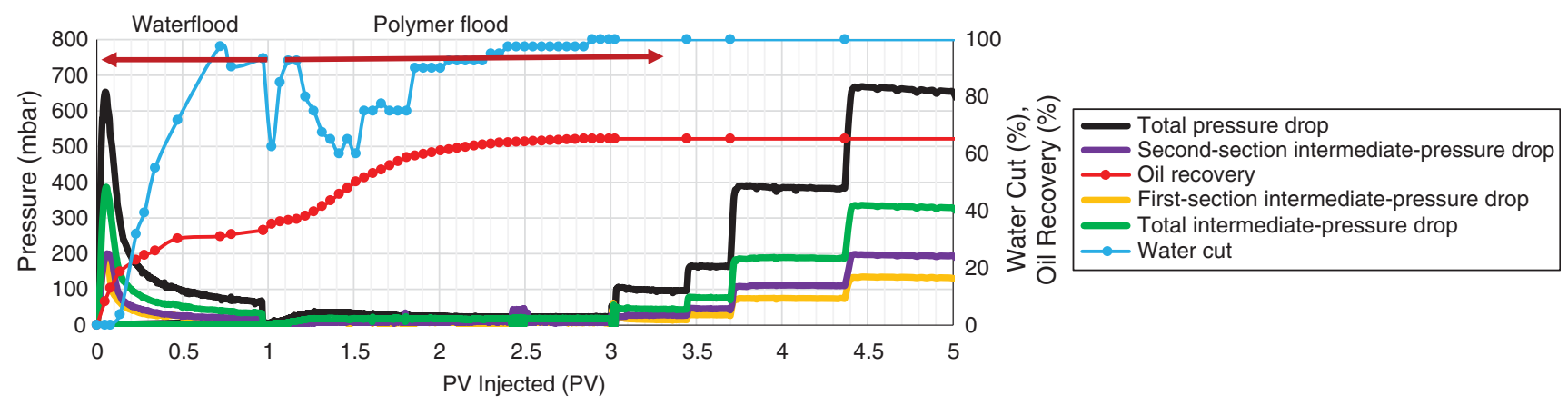

Fig. 8-Waterflood- and polymer-flood-production profiles of WF/PF (1 PV) IWb.

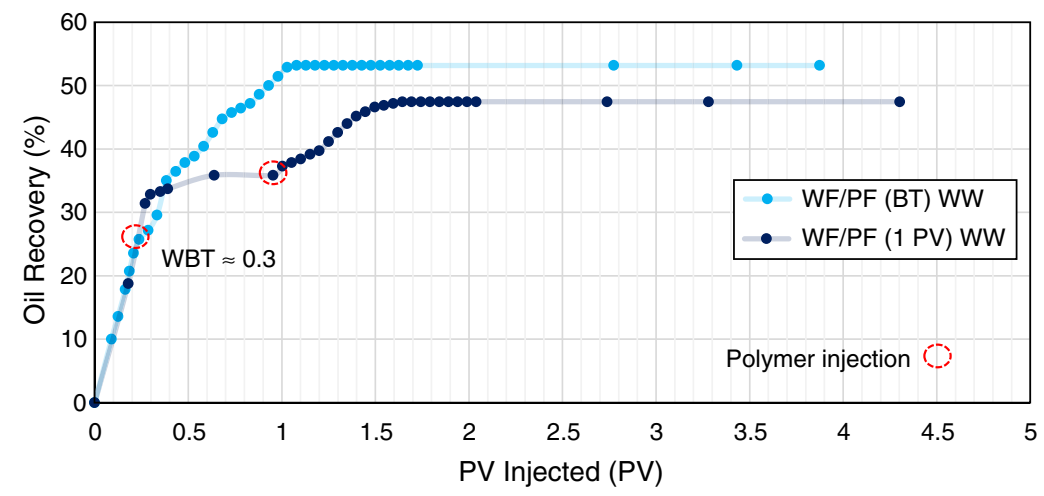

Fig. 9-Oil-recovery curves of experiments performed at different waterflood-maturity times with water-wet cores.

Oil Recovery vs. Maturity Time. Final oil recoveries measured for intermediate-wet and water-wet conditions are summarized in Fig. 10 as a function of injection-maturity times.

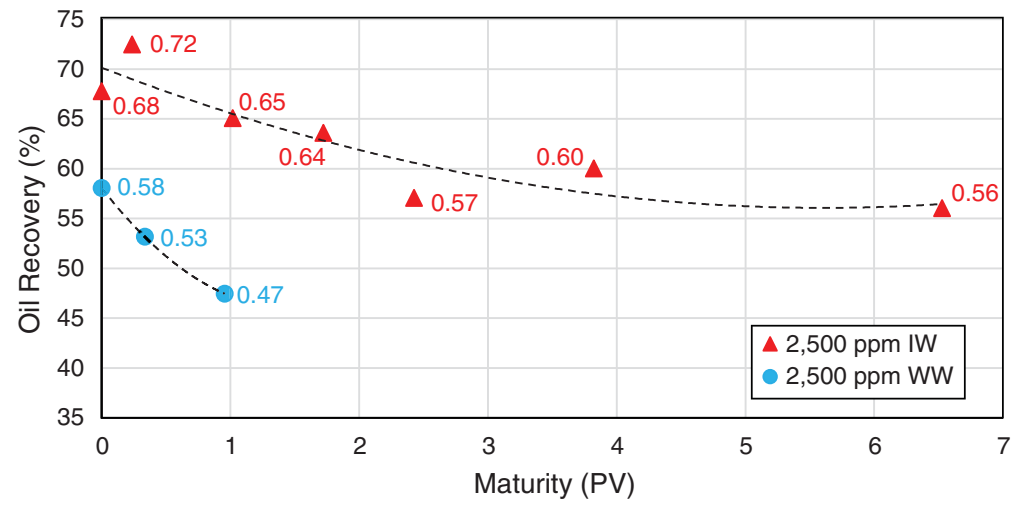

Fig. 10—Final oil recovery vs. maturity time. IW = intermediate-wet; WW = water-wet. 
Considering that all experiments have been performed in mostly the same conditions, with the same capillary number and viscosity ratio but different wettability, we can highlight two main observations.

The first observation concerns the maturity of the polymer injection after a waterflooding. It is clear that the 0-PV maturity time for the polymer flow is the most efficient in terms of oil recovery. For polymer injection after initial waterflooding, the earlier the polymer injection is performed, the higher the final oil recovery.

The second observation concerns the influence of wettability. The polymer-flood final oil recovery for water-wet cores is always lower than the final oil recoveries obtained in the intermediate-wet cores. If the trend is similar, the difference between final oil recoveries seems to increase with waterflood maturity. In contrast, for intermediate-wettability conditions, the final values of oil recoveries do not decrease significantly with water-injection maturity.

Effect of Wettability on Polymer Final Oil Recovery. Waterflood recovery is influenced by wettability, and polymer does not seem to be an exception. It has been reported (Jadhunandan and Morrow 1995) that waterflooding BT occurs later for water-wet cores than for intermediate-wet cores, whereas oil production is almost constant after the WBT for water-wet cores. In contrast, we observe an oilproduction tail for intermediate-wet cores. Final oil recovery during polymer floods after initial waterflooding is always higher for intermediate-wet cores than for water-wet cores.

We observe that the permeability of water $\left(k_{w}\right.$ at $\left.S_{o r}\right)$ at the end of the experiment (see Table 2$)$ is reduced dramatically, and no difference has been observed between $k_{w}$ at $S_{o r}$ values of intermediate-wet cores and water-wet cores. This behavior suggests a possible wettability change during polymer flood, changing the intermediate-wet cores to become water-wet cores. This behavior was observed by Juárez-Morejón et al. (2017).

Mobility Ratio. The mobility ratio $M$ is defined as

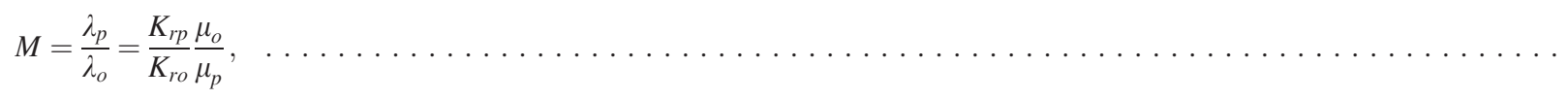

where $\lambda_{p}$ and $\lambda_{o}$ are the polymer and oil mobilities, and $K_{r p}$ and $K_{r o}$ refer to relative permeabilities of the polymer and oil phases. $K_{r p}$ is defined as $\left(k_{p}\right.$ at $\left.S_{o r}\right) / k_{w}$, the last point at constant flow where the oil phase remains immobile. $K_{r o}$ is $\left(k_{o}\right.$ at $\left.S_{w i}\right) / k_{w}$, the value after aging.

The displacement becomes unstable if the mobility ratio $M$ is greater than unity. The higher mobility ratio, the faster instabilities (fingering effects) rise, and consequently the sweep efficiency decreases. Similar values of the final mobility ratio were observed for the different experiments. A similar endpoint mobility ratio is obtained with advanced polymer-injection timing. For the same endpoint mobility ratio (Table 3), different values of oil recovery (Fig. 10) are obtained. We discuss these results next.

Intermediate-Wet

\begin{tabular}{c|ccccccccccc}
\hline Maturity (PV) & 0 & BT & 1 & 1.75 & 2.5 & 4 & 6.5 & 0 & BT & 1 \\
\hline Water saturation & 0.698 & 0.751 & 0.703 & 0.682 & 0.617 & 0.651 & 0.614 & 0.642 & 0.593 & 0.548 \\
Mobility ratio & 6.10 & 4.80 & 5.03 & 8.08 & 6.38 & 7.43 & 4.01 & 2.18 & 4.62 & 3.18 \\
\hline
\end{tabular}

Table 3-Mobility ratio and water saturation.

\section{Discussion}

Several points have to be addressed in the general discussion on the data presented in this paper.

The first point concerns the effect of polymer injection on final oil recovery. The second point, which is less commented on in the literature, is the influence of waterflood maturity on final polymer oil recovery. The final point is the influence of core wettability on polymer-flood incremental recovery.

The experimental results presented in Figs. 4 through 10 show clearly that polymer injection induces in all cases an improved oil recovery compared with waterflooding. This expected result is well-known and is commonly attributed to a more-favorable water/ oil-mobility ratio that is a crucial parameter controlling final oil recovery. For an unfavorable mobility ratio, preferential flow paths can develop and certain zones of the core remain unswept, leading to low oil recovery and early WBT. Viscous fingering is a major cause of low sweep efficiency; it occurs during an unfavorable mobility ratio, and depends on the capillary number and viscosity ratio (Skauge et al. 2014; Shashvat and Mohanty 2015).

Oil-recovery curves plotted in Fig. 11 can be analyzed in terms of oil-production slope, as indicated graphically.

The different recovery-rate values are presented in Fig. 12, where it is observed that the recovery rate $\left[\mathrm{d} R / \mathrm{d}(P V)\left(P V^{-1}\right)\right] \operatorname{decreases}$ when waterflood-maturity time increases. This means that the polymer-solution volume needed to reach the final oil recovery increases with maturity. These data are an indication of the easiness with which the polymer is capable of mobilizing the oil in place (OIP). This ability is linked to the quantity of movable oil available in the core at the moment of polymer injection and the way the fluid phases are dispersed in the porous media.

The influence of polymer maturity on final oil recovery is shown in Fig. 10, where it can clearly be observed that final oil recovery is higher when polymer injection is the earliest. A similar observation can be made from Fig. 12, the trend shape of which is very similar to that observed in Fig. 10. These results suggest that the phase repartition at the pore scale and the water-saturation value at which the polymer injection is started might play an important role in the oil-displacement efficiency. This aspect can be addressed considering two-phase dispersion.

As mentioned in the Experiments section, a dispersion test is performed in the core when it is fully brine saturated $\left(S_{w}=1\right)$. The dispersion curve is obtained from the effluent-density measurement, which is converted into a dimensionless tracer concentration. For some experiments, a second dispersion test was performed at the end of the waterflooding (high water saturation) and at the end of polymer flooding for different maturity times (low and intermediate water saturation). All the dispersion tests were performed at ambient temperature and low flow rate to be sure that the OIP will not move. Examples of dispersion curves are presented in Figs. 13 through 15. 


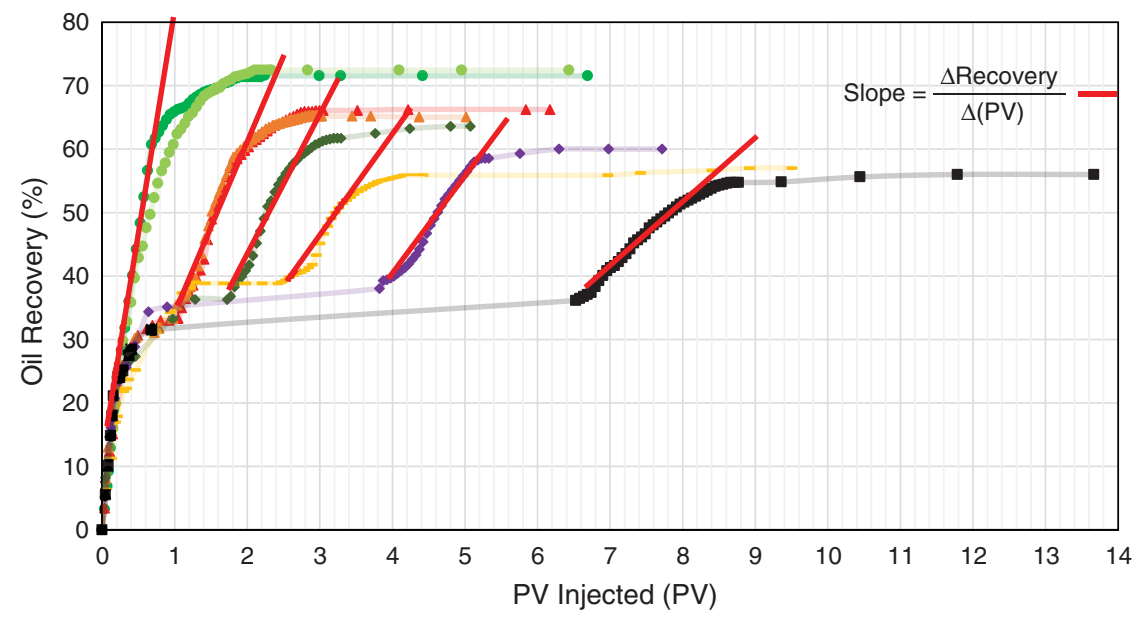

- WF/PF (BT) IWa

$-W F / P F(1.75$ PV) IW

- WF/PF (BT) IWb

- WF/PF (2.5 PV) IW

$\triangle$ WF/PF (1 PV) IWa

- WF/PF (4 PV) IW

$\triangle W F / P F(1 \mathrm{PV}) \mathrm{IWb}$

- WF/PF (6.5 PV) IW

Fig. 11-Oil-recovery slopes.

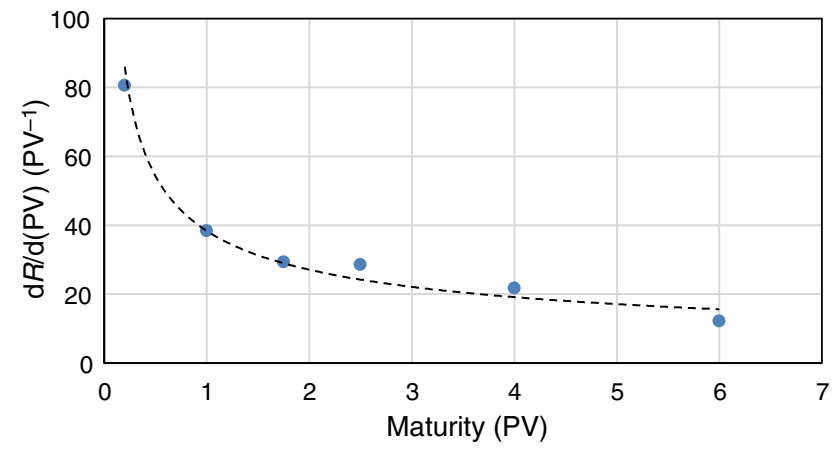

Fig. 12-Oil-recovery rates.

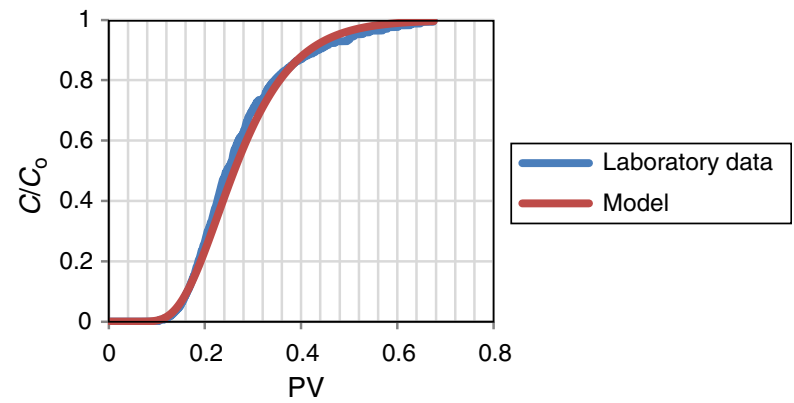

Fig. 13-Experimental data and model fit $\left(S_{w}=0.27\right)$.

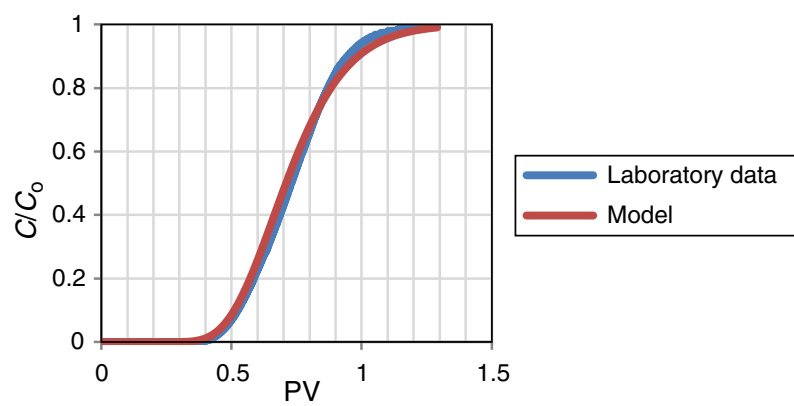

Fig. 14-Experimental data and model fit $\left(S_{w}=0.73\right)$. 


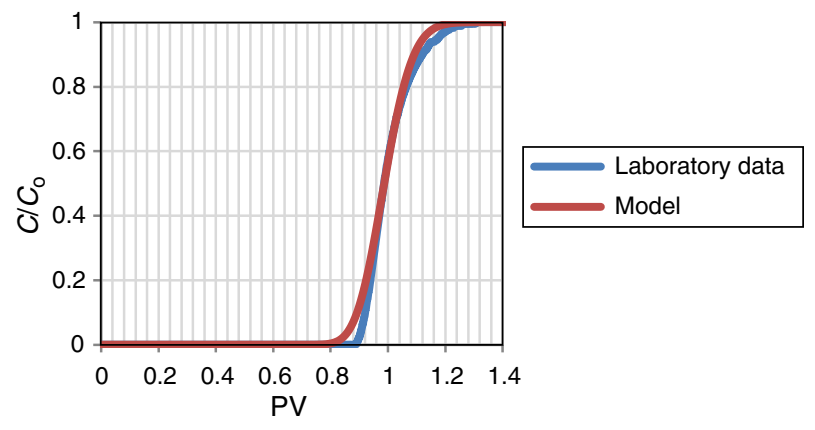

Fig. 15-Experimental data and model fit $\left(S_{w}=1\right)$.

Experimental data are interpreted considering the diffusion/dispersion theory (Runkel 1996) and assuming the 1D analytical solution,

$$
\frac{C}{C_{o}}=\operatorname{erfc}[(x-v t) / 2 \sqrt{D t}]
$$

where $C$ and $C_{o}$ are the effluent and injected-tracer concentrations, respectively; $x$ is the location coordinate; $v$ is the interstitial velocity; $D$ is the dispersion coefficient; and $t$ is time.

All the dispersion experiments were performed at the same Péclet number (Pé of approximately 1),

$$
P e ́=\frac{v d_{g}}{D_{0}},
$$

where $D_{0}$ is the diffusion coefficient $\left(D_{0}=10^{-9} \mathrm{~m}^{2} / \mathrm{s}\right)$ and $d_{g}$ is the porous-medium grain diameter, which can be approximated from the Kozeny-Carman relationship,

$$
k_{w}=\frac{d_{g}^{2}}{180} \frac{\phi^{3}}{(1-\phi)^{2}},
$$

where $\phi$ is the porosity and $k_{w}$ is the permeability of the porous medium.

The dispersion coefficient vs. water saturation is plotted in Fig. 16.

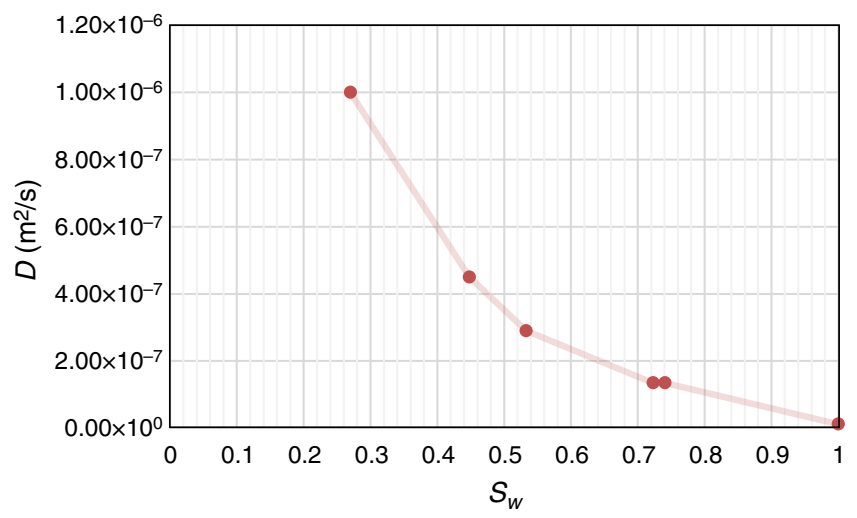

Fig. 16-Dispersion coefficient vs. water saturation.

It is clearly observed in Fig. 16 that the dispersion coefficient decreases when water saturation increases. That behavior is in good agreement with literature results (Thomas et al. 1963). The physical interpretation of this trend is that the water phase is more dispersed in the porous medium when its saturation is low. The history of the water sweep can lead to different distributions of oil/water phases. In the case where the water saturation has been obtained after waterflooding periods with an unfavorable mobility ratio, one can imagine that there are well-established viscous fingers in the porous medium, and that the remaining oil is in unswept zones. In this case, the ability of the polymer solution - with higher viscosity than water-to displace the unswept oil will be difficult because it will flow in the preferential water paths.

When comparing Figs. 10, 12, and 16, we observe a similar trend in terms of final oil recovery, recovery slope, and dispersion coefficient. There is from our point of view a link between all three factors showing that the ability of the polymer to displace the OIP is easier when the water phase is slightly dispersed. The scanning efficiency is related to the ability of the polymer to disperse throughout the accessible pore space. This means that it will be easier for the polymer to mobilize the oil throughout the porous medium, and displace it, if the oil saturation is high.

\section{Conclusions}

From the experimental data presented in this paper, dealing with the influence of polymer-flood maturity and core wettability on the final oil recovery, we note the following conclusions: 
1. A lower remaining oil saturation is achieved with polymer injection compared with waterflooding because of a better oil/polymerviscosity ratio $\left(\mu_{o} / \mu_{p}\right.$ of approximately 10$)$ and a more-favorable mobility ratio.

2. Oil recovery during polymer floods is a strong function of fluid distribution, oil saturation, saturation history, and wettability. Wettability affects polymer flooding by shaping the flow and spatial distribution of oil and water in a porous medium.

3. According to the results obtained in this study, during both waterfloods and polymer floods of a strongly water-wet core, most of the OIP is recovered before BT. After BT, nearly no additional oil is produced.

4. For intermediate-wet systems, the BT of water and polymer floods occurs early, and an important oil volume is recovered after BT. A lower remaining oil saturation is achieved in all cases by the polymer for intermediate-wet conditions compared with waterwet systems.

5. According to our experimental results, an early injection of polymer improves the sweep efficiency at the pore scale.

6. For each waterflood-maturity time there is a different fluid-phase distribution, which results in different dispersion coefficients for each value of water saturation. Our results suggest that the oil phase is more dispersed when the value of the dispersion of the water is lower, causing a decrease of the sweep efficiency of polymer injections.

\section{Nomenclature}

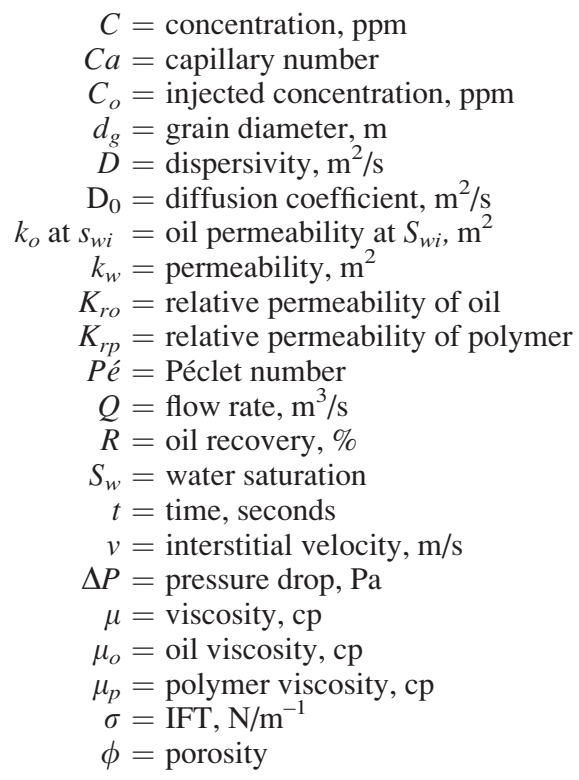

\section{Acknowledgments}

The authors thank Total for supporting this study and for authorization to publish this paper.

\section{References}

Anderson, W. G. 1986. Wettability Literature Survey—Part 1: Rock/Oil/Brine Interactions and the Effects of Core Handling on Wettability. J Pet Technol 38 (10): 1125-1144. SPE-13932-PA. https://doi.org/10.2118/13932-PA.

Asghari, K. and Nakutnyy, P. 2008. Experimental Results of Polymer Flooding of Heavy Oil Reservoir. Presented at the Canadian International Petroleum Conference, Calgary, 17-19 June. PETSOC-2008-189. https://doi.org/10.2118/2008-189.

Buckley, J. S., Liu, Y., and Monsterleet, S. 1998. Mechanisms of Wetting Alteration by Crude Oils. SPE J. 3 (1): 54-61. SPE-37230-PA. https://doi.org/ 10.2118/37230-PA.

Cottin, C., Bourgeois, M., Bursaux, R. et al. 2014. Secondary and Tertiary Polymer Flooding on Highly Permeable Reservoir Cores: Experimental Results. Presented at the SPE EOR Conference at Oil and Gas West Asia, Muscat, Oman, 31 March-2 April. SPE-169692-MS. https://doi.org/ 10.2118/169692-MS.

Jadhunandan, P. P. and Morrow, N. R. 1995. Effect of Wettability on Waterflood Recovery for Crude-Oil/Brine/Rock Systems. SPE Res Eng 10 (1): 40-46. SPE-22597-PA. https://doi.org/10.2118/22597-PA.

Juárez-Morejón, J. L., Bertin, H., Omari, A. et al. 2017. Spontaneous Imbibition as Indicator of Wettability Change During Polymer Flooding. Oral presentation given at the IOR 2017-19th European Symposium on Improved Oil Recovery, Stavanger, Norway, 24-27 April. https://doi.org/10.3997/ 2214-4609.201700334.

Koh, H., Lee, V. B., and Pope, G. A. 2016. Experimental Investigation of the Effect of Polymers on Residual Oil Saturation. Presented at the SPE Improved Oil Recovery Conference, Tulsa, 11-13 April. SPE-179683-MS. https://doi.org/10.2118/179683-MS.

Lake, W. L. 1989. Enhanced Oil Recovery. Upper Saddle River, New Jersey: Prentice Hall.

Levitt, D., Jouenne, S., Bondino, I. et al. 2013. Polymer Flooding of Heavy Oil Under Adverse Mobility Conditions. Presented at the SPE Enhanced Oil Recovery Conference, Kuala Lumpur, 2-4 July. SPE-165267-MS. https://doi.org/10.2118/165267-MS.

Peksa, A. E., Wolf, K.-H. A. A., and Zitha, P. L. J. 2015. Bentheimer Sandstone Revisited for Experimental Purposes. Mar. Petrol. Geol. 67 (November): 701-719. https://doi.org/10.1016/j.marpetgeo.2015.06.001.

Qi, P., Ehrenfried, D. H., Koh, H. et al. 2016. Reduction of Residual Oil Saturation in Sandstone Cores Using Viscoelastic Polymers. Presented at the SPE Improved Oil Recovery Conference, Tulsa, 11-13 April. SPE-179689-MS. https://doi.org/10.2118/179689-MS.

Runkel, R. L. 1996. Solution to the Advection-Dispersion Equation: Continuous Load of Finite Duration. J. Environ. Eng. 122 (9): 830-832. https:// doi.org/10.1061/(ASCE)0733-9372(1996)122:9(830).

Shashvat, D. and Mohanty, K. K. 2015. Fingering Function for Unstable Immiscible Flows. Presented at the SPE Reservoir Simulation Symposium, Houston, 23-25 February. SPE-173290-MS. https://doi.org/10.2118/173290-MS. 
Shiran, B. S. and Skauge, A. 2015. Wettability and Oil Recovery by Polymer and Polymer Particles. Presented at the SPE Asia Pacific Enhanced Oil Recovery Conference, Kuala Lumpur, 11-13 August. SPE-174568-MS. https://doi.org/10.2118/174568-MS.

Skauge, T., Vik, B. F., Ormehaug, P. A. et al. 2014. Polymer Flood at Adverse Mobility Ratio in 2D Flow by X-Ray Visualization. Presented at the SPE EOR Conference at Oil and Gas West Asia, Muscat, Oman, 31 March-2 April. SPE-169740-MS. https://doi.org/10.2118/169740-MS.

Thomas, G. H., Countryman, G. R., and Fatt, I. 1963. Miscible Displacement in a Multiphase System. SPE J. 3 (3): 189-196. SPE-538-PA. https:// doi.org/10.2118/538-PA.

Vermolen, E. C. M., van Haasterecht, M. J. T., and Masalmeh, S. K. 2014. A Systematic Study of the Polymer Visco-Elastic Effect on Residual Oil Saturation by Core Flooding. Presented at the SPE EOR Conference at Oil and Gas West Asia, Muscat, Oman, 31 March-2 April. SPE-169681-MS. https://doi.org/10.2118/169681-MS.

Wang, D., Cheng, J., Yang, Q. et al. 2000. Viscous-Elastic Polymer Can Increase Microscale Displacement Efficiency in Cores. Presented at the SPE Annual Technical Conference and Exhibition, Dallas, 1-4 October. SPE-63227-MS. https://doi.org/10.2118/63227-MS.

Wassmuth, F. R., Green, K., Hodgins, L. et al. 2007. Polymer Flood Technology for Heavy Oil Recovery. Presented at the Canadian International Petroleum Conference, Calgary, 12-14 June. PETSOC-2007-182. https://doi.org/10.2118/2007-182.

José Luis Juárez-Morejón is a post-doctoral-degree researcher in EOR at the University of Bordeaux, France. He holds a PhD degree in mechanics from the University of Bordeaux and a degree in chemical engineering from the University of Valladolid, Spain.

Henri Bertin is a National Center for Scientific Research senior researcher at I2M at the University of Bordeaux. His research interests are fluid mechanics in porous media including EOR processes (polymer, foam) and soil remediation. Bertin holds a PhD degree in mechanical engineering from the University of Bordeaux. He has served as an associate editor for SPE Journal from 2010 to 2018.

Aziz Omari is a professor at Bordeaux-INP, whose research work at the I2M Laboratory is related to various aspects of chemical engineering of dispersed media. He has authored or coauthored more than 100 peer-reviewed papers and conference presentations. Omari holds a PhD degree in the flow of polymer solutions in porous media.

Gerald Hamon has been an expert in petrophysics and oil-recovery mechanisms for more than 20 years at Total. He has authored or coauthored more than 60 technical papers. Hamon holds a PhD degree in fluid mechanics from Grenoble University, France. He is a member of the Society of Core Analysts.

Christophe Cottin is a reservoir engineer with Total. He has 10 years of experience in chemical EOR. Cottin holds a PhD degree from the University of Bordeaux.

Danielle Morel is an EOR expert at Total, based in the Centre Scientifique et Technique Jean Féger of Pau, France. She has worked with the company for more than 20 years, after 10 years as a research engineer at IFP Energies Nouvelles. Morel's main research interests are chemical EOR, miscible processes, and emerging technologies, such as foams and microbial EOR and dealing with laboratory, simulation, and pilot aspects. She holds a master's degree in chemical engineering from the École Nationale Supérieure des Ingénieurs en Arts Chimiques et Technologiques and a PhD degree in thermodynamics. Morel has been an SPE member since 1990 and was an SPE Distinguished Lecturer in 2012-2013.

Carolina Romero is an EOR specialist at Total, based in the Centre Scientifique et Technique Jean Féger. In this role, she currently leads research-and-development (R\&D) activities on EOR deployment and in-depth conformance. Over the last 20 years, Romero occupied different positions in Total and PDVSA Intevep, focused on chemical EOR, single-well chemical-tracer tests, and innovative EOR/improved-oil-recovery technologies using microgels, gels, foams, and nanotechnologies. She holds a PhD degree in chemistry from IFP Energies Nouvelles/University of Besançon, master's degrees in chemical and reservoir engineering, and a bachelor's degree in polymer-material science from Simon Bolivar University, Venezuela.

Gilles Bourdarot has been manager of the EOR R\&D project at Total E\&P since 2014. He has 39 years of experience as a reservoir engineer, holding several R\&D, reservoir-engineering, and management positions with Elf and Total in Pau, the Congo, and Abu Dhabi. Bourdarot has contributed to 20 technical papers, holds two patents, and has published one book (on well-test analysis). He holds an engineering degree from the School of Industrial Physics and Chemistry, France. Bourdarot has been an SPE member since 1999. 